\title{
ATENÇÃO EM VELEJADORES: CONCEITOS E APLICAÇÕES
}

\author{
MS. RICARDO BRANDT \\ Mestre em Ciências do Movimento Humano pela \\ Universidade do Estado de Santa Catarina (UDESC) \\ Pesquisador do Laboratório de Psicologia do Esporte e do Exercício (LAPE) \\ (Florianópolis - Santa Catarina - Brasil) \\ E-mail: ricabrandt@gmail.com \\ MS. MAICK DA SILVEIRA VIANA \\ Mestre em Ciências do Movimento Humano pela \\ Universidade do Estado de Santa Catarina (UDESC) \\ Pesquisador do Laboratório de Psicologia do Esporte e do Exercício (LAPE) \\ (Florianópolis - Santa Catarina - Brasil) \\ E-mail: efisica@gmail.com

\section{MS. LUCIANA SEGATO} \\ Mestre em Ciências do Movimento Humano pela \\ Universidade do Estado de Santa Catarina (UDESC) \\ Pesquisador do Laboratório de Psicologia do Esporte e do Exercício (LAPE) \\ (Florianópolis - Santa Catarina - Brasil) \\ E-mail: lucianasegato@ibest.com.br

\section{DR. ALEXANDRO ANDRADE} \\ Professor do Programa de Pós-Graduação em Ciências do Movimento \\ Humano da Universidade do Estado de Santa Catarina (UDESC) \\ Coordenador do Laboratório de Psicologia do Esporte e do Exercício (LAPE) \\ (Florianópolis - Santa Catarina - Brasil) \\ E-mail: d2aa@udesc.br
}

I. Apoio Financeiro: Ministério de Ciência e Tecnologia - MCT/ Financiadora de Estudos e Projetos - FINEP / Ministério do Esporte - ME. Chamada Pública MCT/FINEP/ME - Ciência e Tecnologia para o Esporte - 01/2006. Processo No 01.06.0633.00. 


\section{RESUMO}

A vela exige de seus atletas conhecimento sobre hidrodinâmica, meteorologia, táticas, além das exigências físicas, técnicas e psicológicas, como a atenção. O objetivo deste estudo foi, por meio de uma revisão de literatura, investigar a atenção e suas implicações na prática da vela. A atenção é uma variável de grande importância no contexto competitivo da vela, sendo uma habilidade fundamental para um bom rendimento. Sugere-se que, para manter níveis adequados de atenção, o atleta estabeleça metas de desempenho, tenha rotinas mentais e faça uso de "palavras gatilho". A atenção está intimamente ligada com as características da vela, podendo ser considerada uma capacidade cognitiva básica para os velejadores.

PALAVRAS-CHAVE: Atenção; concentração; vela; desempenho atlético.

\section{INTRODUÇÃO}

A prática da vela consiste em uma atividade esportiva que, devido às suas complexas características, exige fisicamente do atleta e o estimula intelectualmente (SLATER; TAN, 2007). Um barco a vela sempre navega movimentado pelo vento, pois não é permitido o uso de motores ou outros meios de propulsão durante as regatas (MAIA, 2008). Neste sentido, o deslocamento do barco sobre a água ocorre mediante interação do velejador com as velas, o leme, entre outros equipamentos e forças naturais (água e vento) (MAIA, 2008; BOJSEN-MOLLER; BOJSEN-MOLLER, 1999).

A popularização de esportes náuticos no Brasil, em especial a vela, deu-se ao longo dos anos pelo sucesso dos atletas nacionais em competições ao redor do mundo. Desta forma, pesquisas que investiguem essa modalidade esportiva são importantes para os velejadores e profissionais envolvidos com esse esporte, que podem aproveitar os resultados em suas rotinas de treinamento e competição (RUSCHEL et al., 2009).

A vela é um esporte particularmente interessante do ponto de vista psicológico (ROTUNNO; SENAREGA; REGGIANI, 2004). É uma modalidade que exige movimentos precisos, uma elevada capacidade de concentração e tomada de decisão para antecipar eventos (ROTUNNO; SENAREGA; REGGIANI, 2004; DUARTE; MULKAY; PÉREZ, 2004; ALLEN; JONG, 2006), frente a situações de instabilidade ambiental e imprevisibilidade das condições da competição, o que demanda um adequado controle emocional do atleta.

A atenção é uma habilidade que a pessoa apresenta ao focar em tarefas ou informações externas, bem como em fontes internas, como memória e imaginação. Sendo base para os processos cognitivos, a alteração da atenção implica em dificuldades nas funções psicomotoras, executivas, capacidade de aprendizagem 
e memória (MARTíNEZ-ARÁN et al., 2002). Deste modo, a atenção pode ser considerada fator determinante para um ótimo desempenho atlético, podendo contribuir para melhorar ou prejudicar o desempenho esportivo (MORAN, 2004a; RAGLIN, 200 I), na medida que influencia a cognição e o comportamento do atleta, interferindo decisivamente no processo de tomada de decisão e de execução das habilidades motoras (MACHADO et al., 2009).

Por outro lado, a concentração é a capacidade de manter o foco efetivamente na tarefa, ignorando distrações internas ou externas (MORAN, 2004a). Pelas características da vela, ambas as variáveis, atenção e a concentração, assumem papeis fundamentais na busca pela excelência.

Este trabalho de revisão de literatura tem por objetivo investigar a atenção e suas implicações na prática da vela, apresentando algumas estratégias que podem ser utilizadas pelos velejadores para a melhora de seu rendimento esportivo.

\section{CARACTERÍSTICA E DEMANDAS RELACIONADAS À VELA}

Realizada em um meio pouco usual e normalmente sem contato com o público, a prática da vela requer do atleta boas estratégias, conhecimento teórico de hidrodinâmica, meteorologia, condições de navegação, habilidade de cooperação (em classes com mais de um tripulante) e antecipação de eventos (MAIA, 2008; BOJSEN-MOLLER; BOJSEN-MOLLER, 1999). Nesse contexto, se destaca o velejador que possui um maior conhecimento de ventos e correntes marítimas (MAIA, 2008).

Desde os momentos que antecedem o velejo, o atleta precisa montar e regular o barco conforme as condições climáticas esperadas para a prova. Exige também que ele tome decisões da direção para onde conduzir o barco corretamente na largada, assim como acompanhar visualmente seus adversários, tentando antecipar as ações tomadas por eles. Os velejadores também precisam estar atentos na linha imaginária entre o barco da comissão de regatas e a boia de partida pois, se ultrapassarem esta linha antes do sinal do início da regata, sofrem punição que acarreta em perda de tempo.

O contexto aberto no qual a vela é praticada faz com que cada momento de uma regata seja imprevisível, exigindo constantes adaptações. Durante as regatas (seja em competição ou treinamento), além das preocupações com os adversários, o velejador deve manter as regulagens dos cabos, ajustes da bolina e leme, e posicionamento da escora de maneira a maximizar sua velocidade. Tais procedimentos, em conjunto com suas habilidades para aproveitar efetivamente o vento e a correnteza, proporcionarão um melhor rendimento para o barco. 
Estudos apontam as diversas exigências necessárias para a prática da vela, como: demandas de força, equilíbrio, coordenação, resistência, nutrição adequada, além de boas condições psicológicas para interpretar adequadamente ações do vento e do mar, tomar decisões rápidas, e manter a concentração e o foco de atenção (SLATER; TAN, 2007; DUARTE, 2007; MACKIE; LEGG, 1999; DAUTY et al., 2006; BERNARDI et al., 2007; CUNNINGHAM; HALE, 2007; LÉGER et al., 2007; CASTAGNA; PARDAL; BRISSWALTER, 2007; SPURWAY, 2007; CASTAGNA; BRISSWALTER, 2007; VALLEJO; ROSIQUE; GONZÁLEZ-MORO, 2008). Somado a isso, também se torna necessária uma boa atenção em tarefas individuais e simultâneas, estando estas relacionadas à capacidade do velejador em automatizar tarefas (ABERNETHY et al., 2007).

Durante um dia de competições, é possível ocorrer de uma a três regatas. O intervalo de tempo entre elas pode ser curto ou, dependendo das condições climáticas, muito alongado, ao ponto de atletas passarem horas na água aguardando o início das regatas.

Desta forma, podemos inferir que, ao visualizar as demandas relacionadas à prática da vela durante uma regata, a atenção pode ser fator importante ou fundamental para que o velejador obtenha um ótimo rendimento atlético.

\section{ATENÇÃO EM VELEJADORES}

A atenção é atualmente um dos campos de importância na ciência que investiga e estuda a Psicologia, pois trata de experiências subjetivas e da interação dessas com o comportamento cotidiano (RAZ, 2004). Dentro da Psicologia, a atenção é um conceito paradoxal, mesmo os pesquisadores estando familiarizados com os termos nos mais diferentes contextos e idiomas (ABERNETHY et al., 2007; MORAN, 2009b; VEALEY, 2007). Todos os processos relacionados à atenção estão virtualmente ligados à percepção, cognição e ação, o que torna difícil saber qual aspecto vinculado às habilidades humanas que não é de alguma maneira influenciada pela atenção (ABERNETHY et al., 2007).

Foco de atenção é a habilidade necessária para dirigir e sustentar seletivamente a atenção para uma melhor execução da atividade específica (VEALEY, 2007), derivada de fontes externas ou internas, como memória e imaginação (MORAN, 2004b), envolvendo três aspectos: grande esforço mental, percepção seletiva, e compartilhamento das propriedades mentais. Inclui ainda funções como: manter a atenção por um período, identificar a função de alerta ou vigilância, focalizar apenas os estímulos mais relevantes, entre outras (MATARÓ-SERAT; PUEYO-BENITO; JURADO-LUQUE, 2003). 
Atletas com alto nível de rendimento atlético apresentam aspectos físicos e táticos semelhantes, podendo ser a atenção o fator que proporcionará a vitória ou a derrota. Essa afirmação recebe suporte de Moran (2009a), para o qual atletas de alto nível necessitam de uma atenção efetiva, e isso pode significar um bom ou mau resultado.

Um segundo papel da atenção está relacionado com a capacidade de desenvolver e manter uma ótima sensibilidade e prontidão para responder a estímulos. Em competições esportivas é necessário sustentar ótima atenção por segundos, no caso de um corredor esperando o tiro de partida, ou até mesmo horas, como uma partida de tênis. No caso da vela, o atleta deve estar atendo aos sinais de mudança de vento e maré, e em alguns casos, na mudança de direção de outros competidores.

Observar os demais competidores é uma ferramenta utilizada por velejadores com menor experiência, como aponta o estudo de Araújo, Davids e Serpa (2005). O estudo conclui que a tomada de decisão de velejadores tem relação com o nível de experiência que eles apresentam, onde os velejadores com mais experiência utilizam informações de vento e maré, enquanto os com menos experiência tomam como referência os outros atletas.

Nideffer (1992) afirma que os atletas podem ter quatro tipos de atenção, seja na direção (interna ou externa) ou na amplitude (estreita ou ampla). Além disso, eles devem optar pelo modelo mais apropriado com a demanda exigida em determinada situação esportiva (interno-estreito, interno-amplo, externo-centrado ou externo-amplo). Essas exigências parecem especialmente importantes na vela, onde existe grande variabilidade de focos de atenção, como visto anteriormente.

Moran (2004b; 1996) elaborou cinco princípios teóricos para uma efetiva concentração. Três delas servem para estabelecer atenção, e duas indicam como ela pode estar sendo quebrada:

I. $\bigcirc$ atleta precisa decidir se concentrar. Isso não acontece por casualidade.

2. O atleta só conseguirá a concentração pensando uma coisa de cada vez.

3. A mente do atleta estará no foco quando não houver diferença entre seu pensamento e a tarefa executada.

4. Os atletas perdem a atenção quando o foco de atenção sai do seu controle.

5. Os atletas devem buscar o foco de atenção externo quando ficam nervosos.

De maneira geral, é necessário que o velejador tenha um bom controle da situação e conheça o momento oportuno para iniciar o processo de concentração, utilizando ferramentas previamente elaboradas e treinadas. Focar na tarefa que vai realizar talvez seja uma forma de direcionar o pensamento e conseguir iniciar 
o processo de concentração, o que fará com que diminua a chance de quebrar a atenção.

Neste ponto, é papel do treinador mental, em conjunto com a equipe técnica, trabalhar essas habilidades. No caso da vela, elaborar rotinas para antes das regatas, assim como rotinas utilizadas durantes as mesmas com o intuito de atingir o melhor nível de concentração possível. O companheiro de embarcação (quando em barcos com mais de um tripulante) pode contribuir para que o velejador retome a atenção na tarefa realizada, por meio de um gesto, palavra ou frase positiva.

Poderíamos inferir que cada uma das demandas vistas anteriormente requer uma atenção efetiva por parte do velejador. Em especial nas demandas relacionadas a condições climáticas, onde quebrar o padrão de atenção e, por consequência, tomar uma decisão incorreta ou atrasada, poderia prejudicar de maneira substancial o rendimento na regata.

\section{DESVIO NA ATENÇÃO EM VELEJADORES}

O desvio na atenção se dá por fatores extrínsecos e/ou intrínsecos. Os fatores extrínsecos compreendem as situações do ambiente que desviam a atenção do atleta, como o barulho de multidão, movimentação dos espectadores, condições de tempo, decisões do árbitro, etc. Os fatores intrínsecos são situações subjetivas que incluem o próprio pensamento do atleta, sentimentos e sensações corporais que alteram ou impedem o esforço para se concentrar na tarefa; emoções como raiva e tensão e sensações corporais como dor e fadiga, que impedem o atleta de se concentrar completamente na tarefa (ABERNETHY et al., 2007; MORAN, 2009b).

A pressão na tarefa realizada é um fator intrínseco na distração (BEILOCK; GRAY, 2007). Como na vela os fatores que poderiam causar distração, como o técnico e a torcida, geralmente não estão presentes, a distração causada pela tarefa parece ser o que mais prejudica os atletas. Mas de que maneira isso acontece?

Mesmo estando o atleta altamente concentrado na tarefa, se seu pensamento desvia para outro fator importante, como na preocupação com os adversários (BEILOCK; GRAY, 2007), o foco de atenção se quebra. Isso pode acontecer quando existe a necessidade de mudar a tarefa que está sendo executada, pensando mais no adversário do que nas condições do mar e ventos que vai encontrar na nova direção. Desta forma, é fundamental que o atleta tente manter a concentração na tarefa realizada, deixando fatores externos fora de seu pensamento.

A ansiedade também é fator que influencia na atenção (WILSON; VINE; WOOD, 2009; ABERNETHY et al., 2007). Atletas que apresentam níveis de ansiedade fora da zona ótima, muito elevada ou muito baixa, tendem a perder a 
concentração mais facilmente (BEILOCK; GRAY, 2007). Outro fator é a cegueira por desatenção, que é provocada pela atenção seletiva, que suprime detalhes que podem não ser relevantes para o momento, em favor dos que o são (SIMONS; CHABRIS, 1999). Memmert, Simons e Grimme (2009) citam o exemplo do vídeo onde os observadores tentam contar quantos passes de basquetebol uma equipe de camisa branca realiza, ignorando os passes da equipe de preto. Frequentemente, os observadores não percebem uma pessoa vestida de gorila que passa subitamente pelo vídeo, pois estão dirigindo sua atenção para os passes de maneira seletiva.

Desta forma, percebe-se que os fatores que causam distração acontecem de modo sutil e de forma combinada, quebrando a concentração do atleta (MORAN, 2009b).

\section{DESENVOLVENDO A ATENÇÃO EM VELEJADORES}

Pela importância dos processos de atenção no esporte, uma grande quantidade de estratégias para conseguir a atenção ideal foi estudada e desenvolvida. De maneira geral, essas intervenções acontecem de duas maneiras: através de exercícios e técnicas, que treinem a concentração (MORAN, 2004a). A primeira seria realizada com os atletas em treinamentos, fazendo com que eles treinem sua atenção. A segunda, mais frequente em situações competitivas, com o intuito de colocar o atleta em uma zona ideal de concentração. Nos esportes em geral, inúmeras vezes os atletas são colocados em situações competitivas durante treinamento (MORAN, 2004a).

Talvez um dos primeiros passos para que o atleta atinja e mantenha uma concentração efetiva seja estabelecer metas de desempenho, focalizando em ações e não em resultados. A intenção é fazer com que o atleta focalize em ações ( $M O$ RAN, 2009b). Na vela, um atleta pode focalizar em fazer uma boa largada antes de uma regata, ou seja, estabelecer metas de desempenho, focando em ações e não no resultado final da regata.

A elaboração de rotinas é considerada por Gould, Flett e Bean (2009) uma das melhores ferramentas na preparação mental de atletas. Esta ação consiste basicamente em pensamentos e ações que os atletas devem realizar antes da competição. $\bigcirc$ uso de rotinas psicológicas é importante, pois levam os atletas a pensar e executar a tarefa de modo homogêneo. De modo geral, as rotinas melhoram a concentração, pois ajudam a focalizar em cada tarefa (MORAN, 2009a).

A elaboração de rotinas pré-regata e treinamento mental, bem como a utilização de técnicas de relaxamento, podem proporcionar benefícios aos atletas de qualquer nível competitivo, pois mesmo atletas olímpicos ficam nervosos, se frustram e tem dificuldade na concentração (ALLEN; JONG, 2006). 
Neste contexto, a utilização de palavras "gatilho" ou "palavras chave", entendidas como o uso de lembranças verbais que poderiam ajudar os atletas a focalizar em um objetivo específico ou executar uma ação relacionada, contribui substancialmente para atingir uma concentração efetiva. Poucos estudos testaram a validade das palavras gatilho como técnica na concentração (MORAN, 2004a), porém acredita-se que para ter um efeito positivo é necessário que sejam frases curtas, ilustrativas e vivenciadas positivamente.

A maioria dos atletas fala em tom baixo, quase que secretamente frases como: "que erro estúpido", "o que você está fazendo". No entanto, o propósito do uso de palavras gatilho é ajudar os atletas a re-focalizar na tarefa. Desta forma, os velejadores poderiam usar frases como "presta atenção no seu barco", "concentra na sua regata", "atenção nos seus cabos", fazendo o uso de verbalização de uma maneira positiva, ligada a sua tarefa, pensando em melhorar o desempenho atlético.

Este tipo de técnica colabora com a autoverbalização positiva, a qual influencia positivamente no estado de confiança, motivação e concentração. A autoverbalização pode ser usada em treinamento, ex.: "Hoje treinarei com meu máximo esforço.", "Vou prestar atenção nas instruções do treinador."; antes das regatas, ex.: "Hoje vou fazer uma boa regata."; "Vou tentar manter um concentração ótima durante as regatas."; e durante as regatas, ex.: "Vou ganhar esta regata"; "Vou lutar até o fim.", "Analise as condições e modifique sua estratégia se necessário."; "Confie na sua estratégia.".

Após apresentar algumas técnicas e exercícios para que o velejador melhore a sua atenção, torna-se importante destacar que o profissional que vai aplicar ou orientar alguma dessas técnicas precisa conhecer o atleta, seu conhecimento sobre a modalidade e suas experiências anteriores. Buscando tal compreensão, Moran (2004b) elaborou um questionário que ajuda a entender o que significa "concentração" para o atleta, explorando as estratégias práticas que ele usou para lidar com situações que causaram distração.

Segundo Moran (2004b), normalmente os atletas indicam que a concentração e atenção são realmente importantes para o sucesso no esporte, no entanto, eles raramente dedicam algum tempo em treinamento para melhorar essa habilidade. Cabe ao técnico ou profissional da Psicologia do Esporte alterar essa realidade dos velejadores, haja vista a importância desta variável no contexto esportivo da vela.

\section{CONCLUSÕES}

A atenção está intimamente ligada com as características da vela, podendo ser considerada uma capacidade cognitiva básica em velejadores. No entanto, poucos 
estudos apresentam técnicas utilizadas com o intuito de melhorar ou fazer com que os velejadores encontrem ou atinjam uma zona de atenção ideal.

Em particular, é papel dos treinadores, técnicos e pessoas que trabalham com a Psicologia do Esporte, utilizar estratégias apropriadas para que velejadores atinjam níveis ideais de atenção e concentração.

A vela é um esporte que tem crescido muito em popularidade e competitividade nos últimos anos, tornando necessário técnicas que melhorem o desempenho atlético de seus praticantes. Futuras pesquisas devem aprofundar a relação da atenção com as demandas de desempenho na vela, propondo exercícios e técnicas de concentração específicas para velejadores.

\section{Attention in sailors: concepts and applications}

ABSTRACT: The sail demands from the athlete vast knowledge of hydrodynamic, meteorology, rules and tactics, beyond physical and psychological demands as concentration and attention. The aim of this study was, through a literature review, to investigate the attention and your implication in the practice of sail. Attention is a variable of great importance in the context of competitive sail, considered the necessary ability to a best performance. For the athlete to reach and maintain good levels of attention it is necessary that he establishes performance goals, mental routines and use "trigger words". The attention is intimately linked with the characteristics of sail, and could be considered a basic cognitive capacity for sailors.

KEYWORDS: Attention; concentration; sail; athletic performance.

\section{Atención en velejadores: conceptos y aplicaciones}

RESUMEN: La vela requiere de los atletas conocimientos en hidrodinámica, meteorología, tácticas, además de las capacidades físicas, técnicas y psicológicas, como la atención. El objetivo de este estudio fue, a través de una revisión de literatura, investigar la atención y sus implicaciones en la práctica de la vela. La atención es una variable de gran importancia en el contexto competitivo de la vela, siendo una habilidad fundamental para un gran rendimiento. Se sugiere que para mantener niveles adecuados de atención es necesario que el atleta establezca metas de desempeño, tenga rutinas mentales y hacer uso de "palabras gatillo". La atención es unida íntimamente con las características de la vela, y puede ser considerada una capacidad cognitiva básica para los velejadores.

PALABRAS CLAVES: Atención; concentración; vela; rendimiento atlético.

\section{REFERÊNCIAS}

ABERNETHY, B. et al. Attentional processes in skill learning and expert performance. In: TENENBAUM, G.; EKLUN, R. Handbook of sport psychology. New York: Wiley, 2007. p. 245-263. 
ALLEN, J. B.; JONG, M. R. Sailing and sports medicine: a literature review. British Journal of Sports Medicine, Loughborough, v. 40, n.7, p. 587-593, 2006.

ARAÚJO, D.; DAVIDS, K.; SERPA, S. An ecological approach to expertise effects in decisionmaking in a simulated sailing regatta. Psychology of Sport and Exercise, Amsterdam, v. 6, n. 6, p. 67I-692, 2005.

BEILOCK, S. L.; GRAY, R. Why do athletes choke under pressure? In: TENENBAUM, G.; EKLUN, R. Handbook of sport psychology. New York: Wiley, 2007. p. 425-444.

BERNARDI, M. et al. Physiological characteristics of America's Cup Sailors. Journal of Sports Science, London, v. 25, n. 10, p. I| 4| - | | 52, 2007.

BOJSEN-MOLLER, F; BOJSEN-MOLLER, J. Biomechanics of sailing. In: SJOGAARD, G.; BANGSBO, J. Sailing and science: in an interdisciplinary perspective. Copenhagen: University of Copenhagen, 1999. p. 77-93.

CASTAGNA, O.; BRISSWALTER, J. Assessment of energy demand in laser sailing: influences of duration and performance level. European Journal Applied Physiology, Heidelberg, v. 99, n. I, p. 95-101, 2007.

CASTAGNA, O.; PARDAL, C.; BRISSWALTER, J. The assessment of energy demand in the new olympic windsurf board: neilpryde RS: X. European Journal Applied Physiology, Heidelberg, v. 10, n. 2, p. 247-252, 2007.

CUNNINGHAM, P.; HALE, T. Physiological responses of elite laser sailors to 30 minutes of simulated upwind sailing. Journal of Sports Science, London, v. 25, n. I 0, p. I I 09- I | | 6, 2007.

DAUTY, M. et al. Comparaison de la force isocinétique des rotateurs de l'épaule chez les sportifs de haut niveau pratiquant la voile et les sujets témoins appariés. Science \& Sports, Paris, v. 21, n. 3, p. 154-158, 2006.

DUARTE, R. B. Los estados de ánimo en el deporte: fundamentos para su evaluación. EF Deportes: revista digital, Buenos Aires, v. 12, n. I 10, jul. 2007. Disponível em: <http://mww. efdeportes.com/efd I I0/os-estados-de-animo-en-el-deporte.htm >. Acesso em: I 3 maio 2008.

DUARTE, R. B.; MULKAY, I. Z.; PÉREZ, L. C. Valoración de parâmetros psicosociales em la selección de talentos para el deporte de velas. EF Deportes: revista digital, Buenos Aires, v. I0, n.73, jun. 2004. Disponível em: < http://www.efdeportes.com/efd I I0/los-estados-de-animo-en-el-deporte.htm>. Acesso em: 4 jun. 2009.

GOULD, D.; FLETT, R. M.; BEAN, E. Mental preparation for training and competition. In: BREWER, B. W. Handbook of sports medicine and science. Springfield: Wiley-Blackwell, 2009. p. 53-63.

LÉGER, D. et al. Sleep management and the performance of eight sailors in the tour de France à la voile yacht race. Journal of Sports Science, London, v. 26, n. I , p. 21 -28, 2007. 
MACHADO, N. et al. Transtorno depressivo maior: avaliação da aptidão motora e da atenção. Jornal Brasileiro de Psiquiatria, Rio de Janeiro, v. 58, n. 3, p. 175-180, 2009.

MACKIE, H. W.; LEGG, S. Development of knowledge and reported use of sport science by elite New Zealand Olympic Class Sailors. Applied Human Sciences, Tokio, v. 18, n. 4, p. 125-133, 1999.

MAIA, A. D. Equipamentos utilizados por iatistas da classe snipe: implicações músculo-esqueléticas partir de uma abordagem ergonômica. 2008. I 49 f. Dissertação (Mestrado em Artes e Design) - Pontifícia Universidade Católica do Rio de Janeiro, Rio de Janeiro, 2008.

MARTÍNEZ-ARÁN, A. et al. Neuropsychological performance in depressed and euthymic bipolar patients. Neuropsychobiology, Basel, v. 46, n. I, p. 16-21, 2002.

MATARÓ-SERRAT, M. M.; PUEYO-BENITO, R. O.; JURADO-LUQUE, M. A. Rehabilitación de la atención. Avances en Psicologia Clinica Latinoamericana, Bogota, v. 2 I , p. 3 I -38. 2003.

MEMMERT, D.; SIMONS, D. J.; GRIMME, T. The relationship between visual attention and expertise in sports. Psychology of Sport and Exercise, Amsterdam, v. I 0, n. I, p. I 46- I 5 I , 2009.

MORAN, A. Cognitive psychology in sport: progress and prospects. Psychology of Sport and Exercise, Amsterdam, v. I 0, n. 4, p. 420-426, 2009 a.

Attention and concentration training in sport. In: SPIELBERGER, C. Encyclopedia of Applied Psychology. New York: Academic Press, 2004a. p. 209-2I4.

Attention, concentration and thought management. In: BREWER, B. W. Handbook of sports medicine and science. Springfield: Wiley-Blackwell, 2009b. p. I 8-29.

MORAN, A. Sport and exercise psychology: a critical introduction. London: Psychology Press, 2004b.

The Psychology of concentration in sport performers: a cognitive analysis. London: Psychology Press, 1996.

NIDEFFER, R. M. Psyched to win. Champaign: Leisure, 1992.

RAGLIN, J. S, Psychological factors in sport performance: the mental health model revisited. Sports Medicine, Auckland, v. 31, n. I2, p. 875-890, 2001.

RAZ, A. Attention. In: SPIELBERGER, C. Encyclopedia of applied psychology. Tampa: Elsevier, 2004. p. 203-208.

ROTUNNO, R.; SENAREGA, D.; REGGIANI, E. Psychological support in top level sailing. International Journal of Sport Psychology, Rome, v. 35, n. I, p. 13-22, 2004.

RUSCHEL, C. et al. Incidência de lesões em velejadores brasileiros de diferentes níveis técnicos. Revista Brasileira de Medicina do Esporte, São Paulo, v. I 5, n. 4, p. 268-27I , 2009. 
SIMONS, D.; CHABRIS, C. Gorillas in our midst: sustained inattencional blindness for dynamic events. Perception, London, v. 28, n. 9, p. 1059-1074, 1999.

SLATER, G.; TAN, B. Body mass changes and nutrient intake of dinghy sailors racing. Journal of Sports Science, London, v. 25, n. I 0, p. I I29- I 135, 2007.

SPURWAY, N. C. Top-down studies of the genetic contribution to differences in physical capacity. In: .; WACHERHAGE, H. Genetics and molecular biology of muscle

adaptation. Churchill Livingstone: Elsevier, 2007. p. 25-59.

VALLEJO, J. M. B.; ROSIQUE, D. F.; GONZÁLEZ-MORO, I. M. La fuerza exlposiva em regatistas de la clase tornado. EF Deportes: revista digital, Buenos Aires, v. 13, n. I 19, abr 2008. Disponível em: <http://www. efdeportes.com/efd I 19/la-fuerza-explosiva-en-regatistas-de-la-clase-tornado.htm>. Acesso em: 4 jun 2009.

VEALEY, R. S. Mental skills training in sport. In: TENENBAUM, G.; EKLUND, R. C. Handbook of sport psychology. New Jersey: Wiley \& Sons, 2007. p. 287-30I.

WILSON, M. R.; VINE, S. J.; WOOD, G. The influence of anxiety on visual attentional control in basketball free throw shooting. Journal of Sport \& Exercise Psychology, Champaign, v. 31, n. 2, p. 152-168, 2009.

Recebido: 16 ago 2010

Aprovado: 13 jul 201 |

Endereço para correspondência:

Ricardo Brandt

Centro de Ciências da Saúde e do Esporte Laboratório de Psicologia do Esporte e do Exercício Rua Pascoal Simone, 358 - Coqueiros

Florianópolis-SC CEP: 88080-350 